<smiles>CCC1(C)OC2(C)CC1C2(O)Cl</smiles>

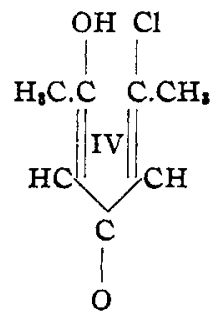

All of them have been discussed and some of them defended, but surely not one of them can account for electrolytic dissociation, so that the oxonium formula must be correct:<smiles>CC1(C)C=CC(=O)C=C1Cl</smiles>

I am going to extend these investigations to the addition compounds of ethers with acids. The simple structure of the ethers will entail that the structure of the addition compounds is quite doubtless, if they turn out to be salts like dimethylpyronehydrochloride.

polytechiscal Higz School,

Copenhagen, Denmark.

\title{
THE PRODUCTION OF CHLOROPICRIN BY THE ACTION OF AQUA REGIA ON ORGANIC COMPOUNDS. \\ [PRELIMINARY PAPER.]
}

By Rasix Lal Datta and Nihar Ranjan Chatrerjee.

Received January 12, 1915.

Aqua regia has been found to be a chlorinating ${ }^{1}$ as well as an oxidizing agent. ${ }^{2}$ It has now been found that it sometimes acts destructively, leading to the rupture of organic substances subjected to its action, with the production of chloropicrin. The transformation into chloropicrin is quantitative in some cases, in others it takes place to a limited extent while in some cases it does not take place at all. The action of aqua regia on the following substances has been studied.

Acetone.-Aqua regia decomposes acetone almost quantitatively into chloropicrin and the reaction can therefore be employed as the best method

${ }^{1}$ Datta and Fernandes, This Journal, 36, 1007 (19r4).

${ }^{2}$ Datta, Ibid., 36, Ior I (1914). 
for the laboratory preparation of this substance. Hofmann ${ }^{1}$ gave a laboratory method for the preparation of this compound by the action of bleaching powder on pictic acid. This procedure, however, gives only poor yields and is troublesome to carry out.

To a mixture of 2 parts of nitric acid and 3 parts of hydrochloric acid, acetone is gradually added, the acid mixture being warmed slightly. An oil separates at first, which soon dissolves with the production of the characteristic odor of chloropicrin. The addition of acetone is stopped when a quantity equal to a tenth part of the acid mixture has been added. When the whole of the acetone has been added the mixture is warmed on the water bath for some time to enable the reaction to become complete. The resulting liquid is next subjected to steam distillation and the compound is separated, dried by means of calcium chloride and finally distilled at a slightly reduced pressure.

The substance boiled between $\mathrm{II} 3^{-114^{\circ}}$ at about $75^{\circ} \mathrm{mm}$. The yield obtained was $85 \%$ of the theory. To establish its identity a chlorine determination was made.

0.166I gave $0.4288 \mathrm{AgCl}$. Calc. for $\mathrm{CCl}_{3} \mathrm{NO}_{2}: \mathrm{Cl}, 64.74$; found: 63.86 .

It has been observed that if the reaction is carried out in the cold, with very gradual addition of acetone, an oil containing chloropicrin separates, which, on slow evaporation in a desiccator, yields colorless crystals having m. p. $103-104^{\circ}$. This is being investigated.

Allyl Alcohol.-The action of aqua regia on allyl alcohol was next studied, in as much as it contains an unsaturated bond. When the reaction was carried out with warming, quantitative production of chloropicrin was the result; but when it was carried out in the cold, a mixture of chloropicrin and another substance which is under investigation was produced. Allyl alcohol was gradually added to a mixture of the acids $(2: 3)$, with occasional warming on the water bath. Its transformation into chloropicrin was complete. The resulting solution was subjected to steam distillation. A colorless oil was obtained which was identified by distillation as usual.

Ether.-When ether was added in small portions to aqua regia no change was apparent at first, but on warming limited action took place. At the end of the reaction no oil was deposited, a pungent odor of chloropicrin being noticed as usual. The product was separated by steam distillation and it was found that a very small quantity of chloropicrin had been formed.

Ethyl Alcohol.-Ethyl alcohol is decomposed only partially with the formation of chloropicrin. To a mixture of nitric and hydrochloric acids (2.3), ethyl alcohol was added in small quantities. At first no reaction took place, but after a time the action became violent and the

I Ann., I39, I I I (I 866). 
acid mixture had to be cooled. After the action had subsided the mixture was warmed on the water bath for a few minutes. The chloropicrin was recovered as usual after steam distillation.

Methyl Alcohol.-Methyl alcohol remains undecomposed by aqua regia even on warming on the water bath and hence no chloropicrin is produced. After a very prolonged action only a slight smell of chloropicrin could be noticed.

Formic and Acetic Acids.-These acids are quite stable and remain undecomposed even on warming. Consequently no chloropicrin is formed in these cases.

A host of other substances has been found to give chloropicrin as the ultimate decomposition product, and it appears that the production of chloropicrin might be quite general. Investigations to establish this as well as a general study of the action of aqua regia and also the action of nitric acid in conjunction with bromine and iodine on organic bodies are being continued.

\footnotetext{
Chemical, Laboratory,

Presidency Collegge, Calcutta.
}

\section{CONTRIBUTIONS TO THE KNOWLEDGE OF HALOGENATION. VII. ${ }^{1}$ SUBSTITUTED NITROGEN CHLORIDES. THE ACTION OF CHLORINE ON CARBAMIC ESTERS AND BIURETS AND THE PREPARATION OF CHLOROCAR- BAMIC ESTERS AND CHLOROBIURETS. \\ By Rasty Lai Datta and Satyaranjan das Gupta. Received January 12, 1915.}

The action of chlorine on ethyl carbamic ester has been studied by $\mathbf{u s}^{2}$ with the isolation of monochlorocarbamic ester. Schmidt ${ }^{3}$ obtained a condensation product of urethane with dichloroacetaldehyde, viz., dichloro-ethylidene urethane by the action of the same reagent on the ester at a high temperature.

It has been found that the chloroderivatives of other esters of the series can be prepared by working with aqueous solutions of the urethanes in the cold. These have been specially isolated since they might serve as useful synthetic reagents on account of their greater stability than similarly substituted carbamide derivatives. It has been noted under monochlorocarbamic ester, that it readily serves for the chlorination of amines and amides which hitherto had to be chlorinated by means of alkaline

${ }^{1}$ Previous communications on the subject; see Datta, J. Chem. Soc., ror, I66 (Igr2); Datta and Ghosh, Thrs Journal, 35, IO44 (I9I3); Datta and Gupta, Ibid., 36, 386 (I914); Datta and Fernandes, Ibid., 36, I007 (1914); Ibid., 36, IOII (I9I4); Datta, Ibid., 36, Ior I (19r4).

${ }^{2}$ Datta and Gupta, Thrs Journal, 36, 386 (I9I4).

${ }^{3}$ Schmidt, J. prakt. Chem., [2] 24, I 20 (1881). 